\title{
Review Paper \\ Systematic Review and Meta-analysis of Health Literacy in Iranian Older Adults
}

\author{
Majid Mirmohammadkhani ${ }^{1} \odot$ Abbas $^{\text {Ziari }}{ }^{1} \odot{ }^{\circ}{ }^{*}$ Marjan Momeni $^{2} \odot$
}

1. Social Determinants of Health Research Center, Semnan University of Medical Sciences, Semnan, Iran.

2. Department of Health Information Technology, School of Rehabilitation, Semnan University of Medical Sciences, Semnan, Iran.

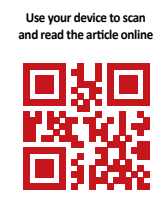

Cteation: Mirmohammadkhani M, Ziari A, Momeni M. [Systematic Review and Meta-analysis of Health Literacy in Iranian Older Adults (Persian)]. Iranian Journal of Ageing. 2020; 15(1):2-13. https://doi.org/10.32598/sija.2020.3.210

doi https://doi.org/10.32598/sija.2020.3.210

Key words:

Health literacy, Elder-

ly, Systematic review,

Meta-analysis, Iran

\section{A B S T R A C T}

Objectives Health Literacy is the capacity of individuals to obtain, interpret, and understand health information for appropriate decision making. Elderly self-care is a supportive strategy in many diseases that requires a high level of health literacy. The aim of this study was to determine the level of health literacy of Iranian elderly people based on scientific evidence.

Methods \& Materials The search was carried out in international and regional scientific databases as well as Google Scholar, using the keywords "health literacy," "aging", and "Iran" in Persian and English, until 2018 October. Totally 321 titles were retrieved. After screening, the full text of 13 articles on the health literacy of Iranian older adults was examined. Finally, the information of 6 articles which was qualitatively appropriate and relevant to the criteria for entering the study, were included in the meta-analysis. Metaanalysis was performed by using STATA software.

Results The mean score of health literacy in the elderly is 45.8 and the estimated $95 \%$ confidence interval is 36.05 to 55.55 . Based on the results of repeated meta-analysis in subgroups (based on gender-disaggregated data), the average health literacy score in male population is higher than that of women (57.24 vs 44.28).

Conclusion The results of this study showed that the Iranian older adults have a relatively low level of health literacy. Considering the importance of health literacy and its impact on the quality of life of this population, Planning and effective interventions to improve the health literacy of the Iranian elderly are needed.

\section{Extended Abstract}

\section{Introduction}

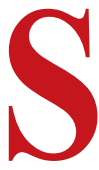

elf-care and accountability of the elderly against various diseases require an acceptable level of health literacy [1]. Health literacy is defined as the degree to which individuals have the capacity to acquire, interpret, and understand health information and services that are necessary for appropriate decision making [2]. In a report, the World
Health Organization introduced health literacy as one of the biggest determinants of health and advised countries around the world to monitor and coordinate strategic activities to promote health literacy in their community [3].

The population of the elderly in Iran is increasing [4]. Elderly people with poor health literacy are more likely to develop chronic illnesses, to see a doctor, and to need more emergency care [5]. They are at a lower level of health, have a misunderstanding of health information, and seek medical help when their problem has reached a critical state

\section{* Corresponding Author:}

Marjan Momeni, PhD \& MD.

Address: Social Determinants of Health Research Center, Semnan University of Medical Sciences, Semnan, Iran

Tel: +98 (23) 33654185

E-mail: mmomeni386@gmail.com 
[6]. Inadequate health literacy is associated with inappropriate use of medications, failure to follow a doctor's instructions, and prolonged illness [7].

In Iranian society, several studies have been conducted to assess the level of health literacy of the elderly and its determinants. Considering that these studies were each conducted in a geographical area and on a limited population of Iran's elderly people, a systematic search and preparation of a more accurate report using meta-analysis of available data seemed necessary. It is obvious that by summarizing the obtained information, a better basis is provided for the knowledge of experts and decision makers in the field of aging so that they can decide on determining the health level and promoting it in such a way that the elderly can enjoy a better quality of life. This study aimed to retrieve and summarize the previous studies and was conducted in order to more accurately assess the level of health literacy of Iranian elderly in the form of a systematic review and meta-analysis.

\section{Methods \& Materials}

According to the registered protocol of this study with the number CRD42018098934 on the PROSPERO website, in the first stage, the search and retrieval of articles and studies related to the health literacy of the elderly in Iran was performed. PubMed (Medline), Science Direct, Web of Science, ProQuest and Scopus databases, as well as three Persian language databases named SID.ir, Irandoc.ir, and Magiran.ir were searched by the scientific search engines scholar.google.com and Elmnet.ir (until October 2018).

The keywords "health literacy, HL", "Iran", "older adults", "elderly", "aged", and "senior, aging" were searched in both Persian and English to retrieve articles. Search strategy, screening and data selection were performed according to PRISMA criteria. Theses and abstracts of conference papers that could not be accessed in full were excluded.

In the next step, after checking duplicate contents, the titles and abstracts of the retrieved studies were independently reviewed by two researchers so that only studies that met the inclusion criteria were identified and entered into the study. In case of any disagreement, a final agreement was reached with the participation and opinion of a third party. Then, the full text of all studies that met the inclusion criteria was retrieved. If in one study the level of health literacy of the elderly was not expressed in numbers, that article was excluded from the study. Regarding the age range of participants, if the title, abstract, implementation method or study objectives explicitly referred to the elderly group as the target group, the article was included in the study.
Evaluation and confirmation of the quality of the studies were performed using the STORB checklist. Then, the data including the studied city, survey tool, study type, number of samples, average health literacy in general and also by gender (if there was a report) were extracted from selected articles and recorded in Excel software. STATA-11 software was used for meta-analysis. To examine the publication bias, a funnel diagram was drawn based on the data, taking into account the sex variable.

To check the heterogeneity, I-square index was used (values above 50 heterogeneities were considered). Data from each of the male and female population groups, if available, were entered separately in the main meta-analysis and subgroup analysis process. The meta-analysis was performed using the fixed effects model and a separate forest plot was drawn for each analysis.

\section{Results}

After reviewing the retrieved articles and evaluating the quality of the articles, 6 articles were meta-analyzed. These articles were published between 2012 and 2018, and the research method was cross-sectional. The sample size in these studies was 1655 people whose data were analyzed.

The "Test of Functional Health Literacy in Adults" (TOFHLA) was used to measure health literacy in all studies. Only one study used the Short TOFHLA (S-TOFHLA) tool. The mean scores of health literacy in each of the studies and also the pooled mean scores with the CI of $95 \%$ in the elderly population of Iran can be seen in the forest plots (Figure 1).

Total mean score of health literacy was 45.8 , and the $95 \%$ confidence interval was estimated between 36.05 and 55.55. Based on the results of repeated meta-analysis in the subgroups, it was observed that the average score of health literacy in the population of elderly men was higher than women (57.24 vs. 44.28).

\section{Conclusion}

The results of this study showed that Iranian elderly have relatively low health literacy and due to the importance of health literacy and its impact on various indicators of the elderly lives, there is a need for effective planning and interventions to promote health literacy in the elderly. Limited health literacy is one of the health-related challenges and a major health concern for older people, as they often need adequate health information to maintain and improve their health. 


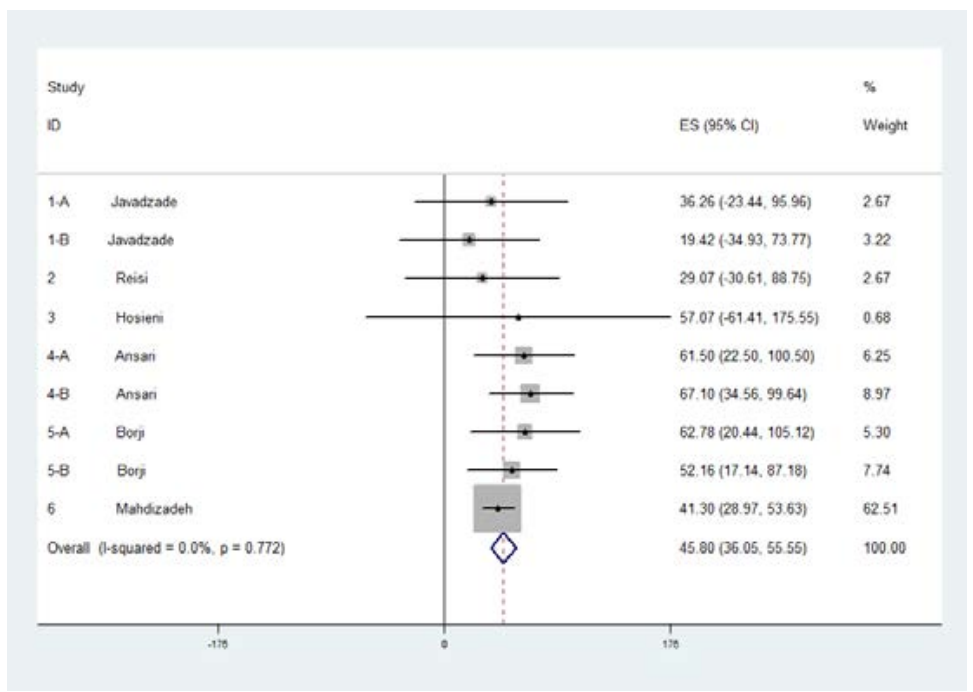

Figure 1. Forest plot of the means of health literacy scores

According to the results of the present study, the health literacy of elderly women is lower than that of men, and therefore it seems that interventions to increase the health literacy of elderly women is a priority.

Our country is going to face an increase in the elderly population in the coming years, and therefore health literacy can be effective in improving the quality of life of the elderly, controlling and self-management of the disease in them, reducing referrals to health centers and reducing health system costs. Therefore, it is inevitable to pay attention to issues that affect the health literacy of the elderly. Our suggestion to specialists and researchers in the field of aging is that in their future research on the effectiveness of methods and programs related to promoting health literacy in the elderly community, they pay attention to issues such as culture building and production of comprehensible educational materials.

\section{Ethical Considerations}

Compliance with ethical guidelines

This study was approved by the Ethics Committee of Semnan University of Medical Sciences (IR.SEMUMS.REC.1397.045).

\section{Funding}

This study received funding from the Vice Chancellor for Research and Technology of Semnan University of Medical Sciences.
Authors' contributions

All authors were equally contributed in preparing this article.

\section{Conflicts of interest}

The authors declared no conflict of interest.

\section{Acknowledgements}

We would like to thank the Clinical Research Development Unit of Amiralmomenin Educational and Research and Therapeutic Center of Semnan University of Medical Sciences for their cooperation. 


\title{
مرور سيستماتيك و مثاآناليز سواد سلامت در سالمندان ايران
}

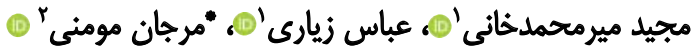 \\ 1. مركز تحقيقات عوامل اجتماعى مؤثر بر سلامت، دانشكاه علوم يزشكى سمنان، سمنان، ايران.

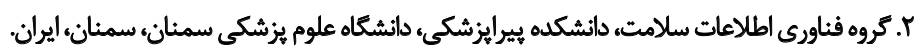

\begin{abstract}
حكSد

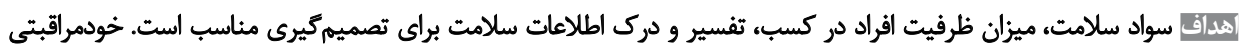

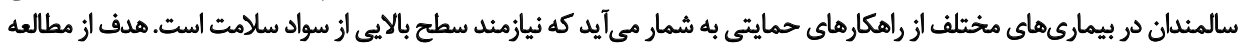

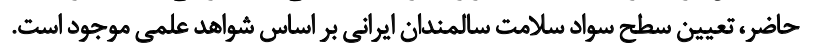

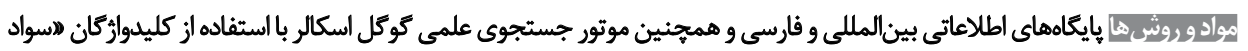

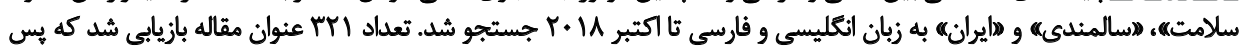

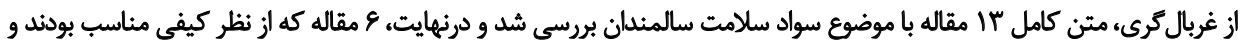

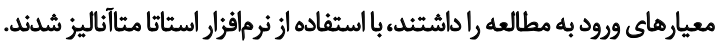

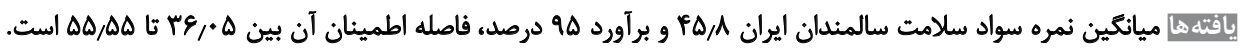

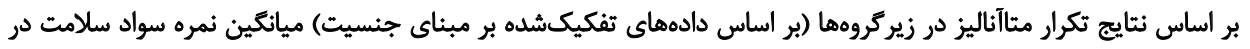

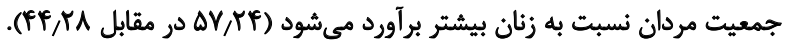

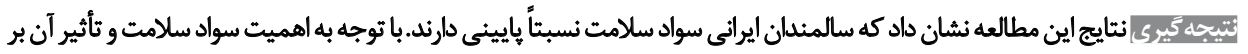

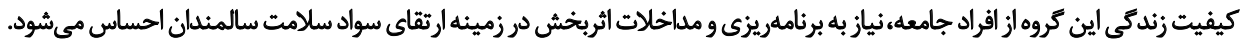

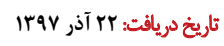

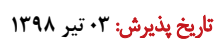

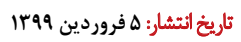

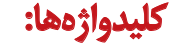

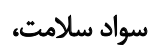

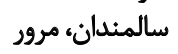

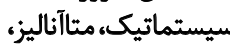 ايران}

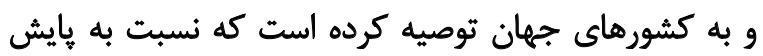

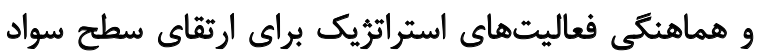

مقدمه

سلامت در جامعه خود اقدام كنيند [9].

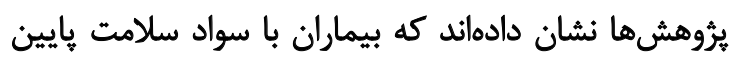

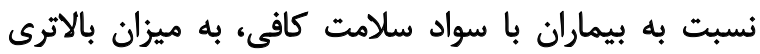

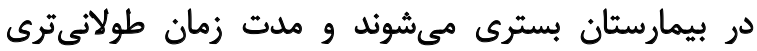

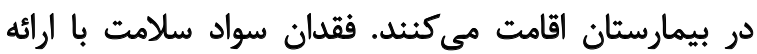

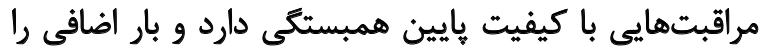

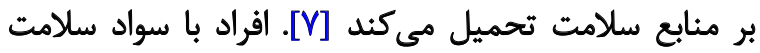

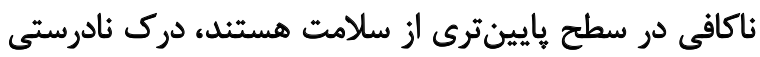

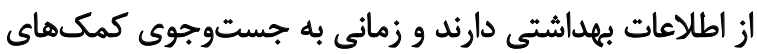

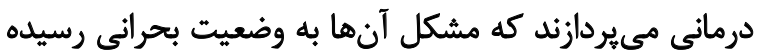

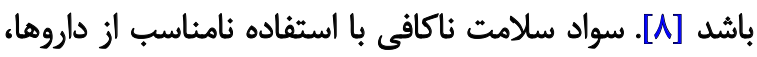

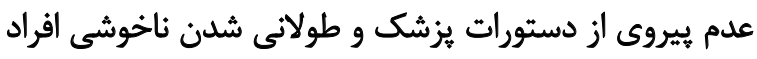
در اغلب جوامع، سالمندان با بيشترين خطر كاهش

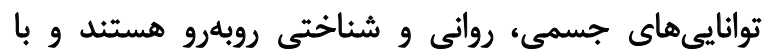

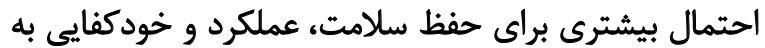

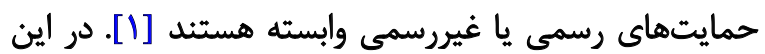

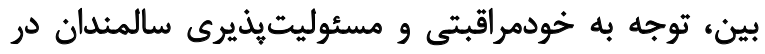

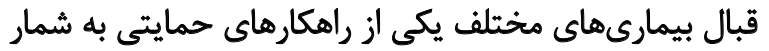

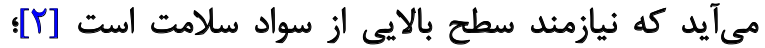

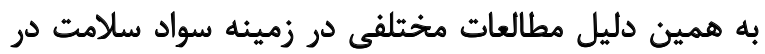

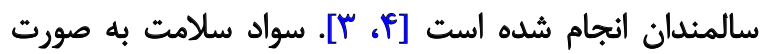

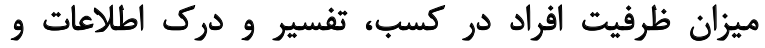

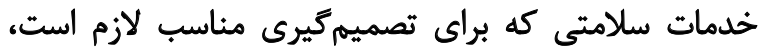

تعريف شده است [ه].

همراه است [9].

در سالمندان نيز وضعيت سلامت عمومى و خودكارآمدى با

سازمان بهداشت جهانى در تزارشى، سواد سلامت را در رديف يكى از بزركترين تعيين كنندهان ساى سلامت معرفى كرده

\section{-}

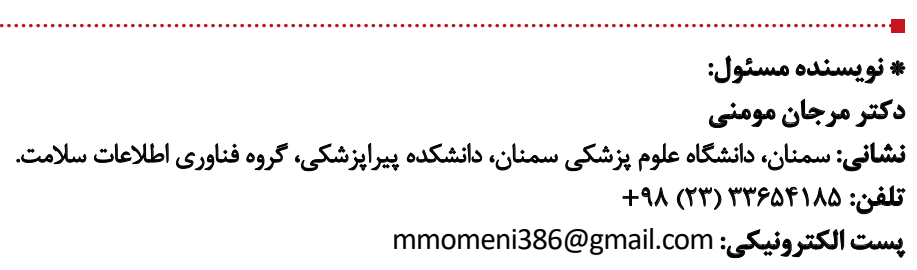


dوأ

يرويوزال اين يُروهش با كد

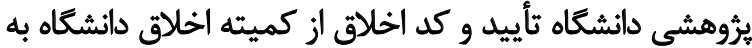

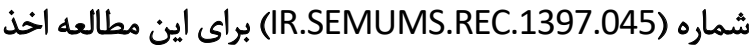

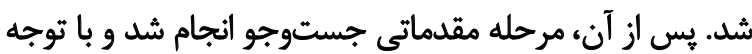

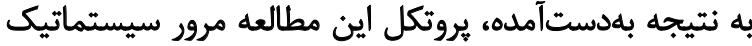

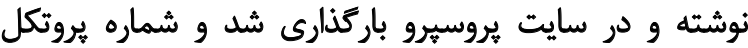

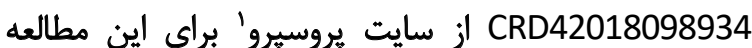

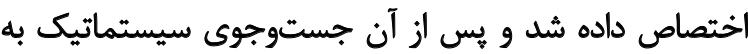
شرح زير انجام شد: - ماده

استراتثى جستوجو: به منظور دسترسى به مطالعات مرتبط

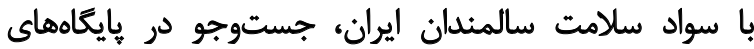

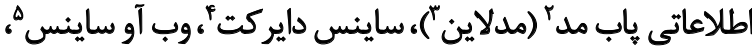

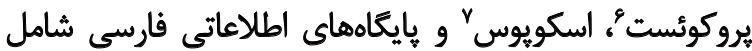

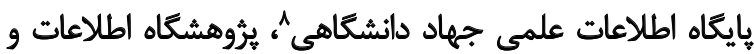

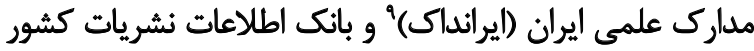

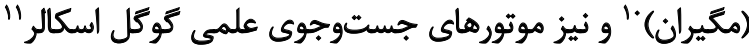

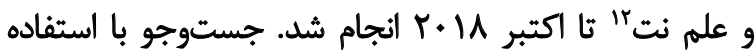

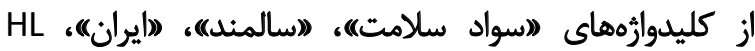
“Health literacy» «Iran» «Older adults» “"Elderly»

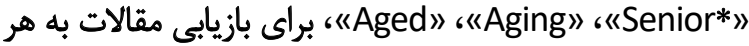

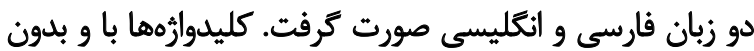

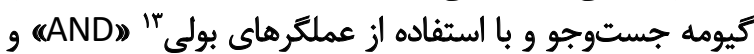

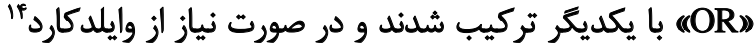

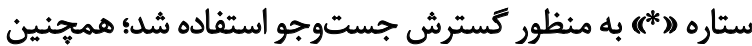

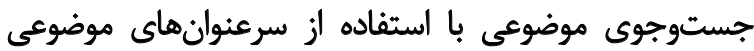
يزشكى MeSH وهاز واز طريق بانك اطلاعاتى يابمد (مدلاين) انجام

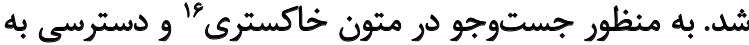

\section{PROSPERO}

2. PubMed

3. Medline

4. Science Direct

5. Web of Science (WOS)

6. ProQuest

7. Scopus

8. SID.ir

9. Irandoc.ir

10. Magiran.ir

11. Scholar.google.com

12. Elmnet.ir

13. Boolean operators

14. Wildcard

15. Medical subject heading

16. Gray literature
سواد سلامت ارتباط دارد [•1]]. سالمندانى كه سواد سلامت بـإيت

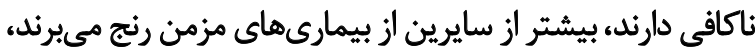

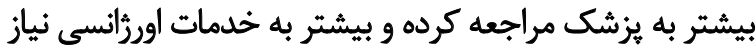

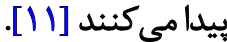
جمعيت افراد سالمند در كشور ايران در حال افزايش است.

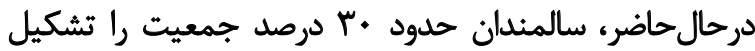

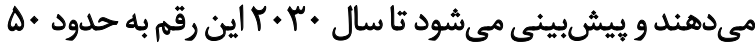

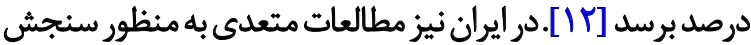

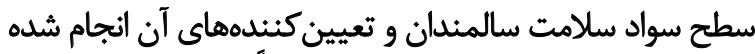

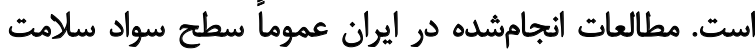

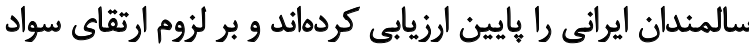

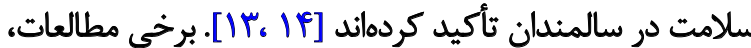

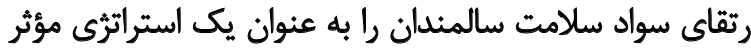

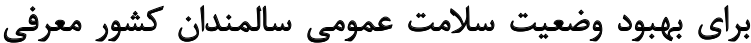

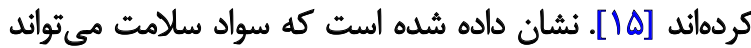

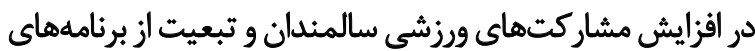

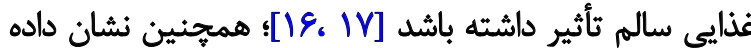

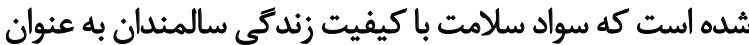

يك شاخص تعيينكنئده و اساسى ارتباط دارد [19، 19] توجه به سواد سلامت براى ارتقاى سلامت سالمندان ضرورى

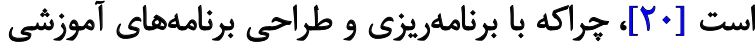

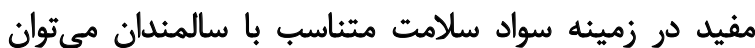

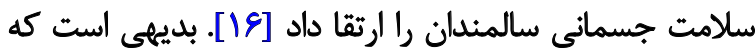

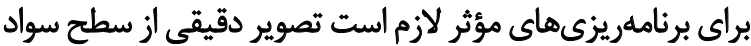
سلامت سالمندان در ايران داشته باشيهم.

با توجه به اينكه مطالعات انجامشده در مورد سواد سلامت

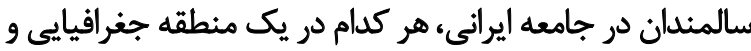

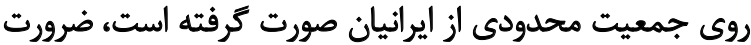

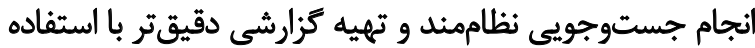

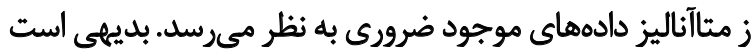

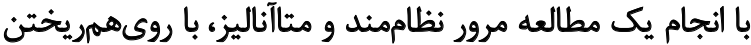

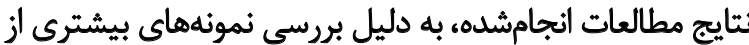

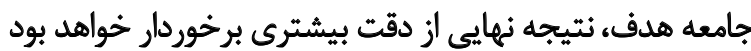

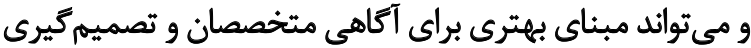

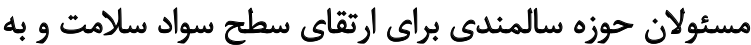

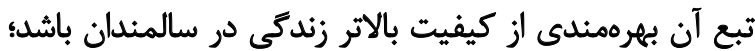

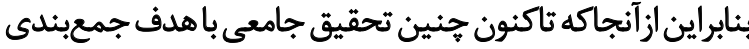

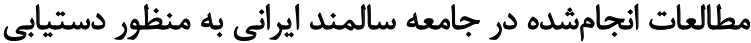

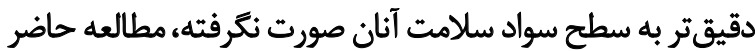

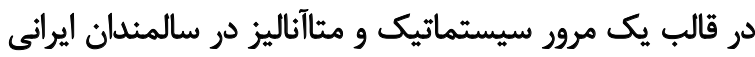

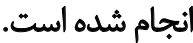



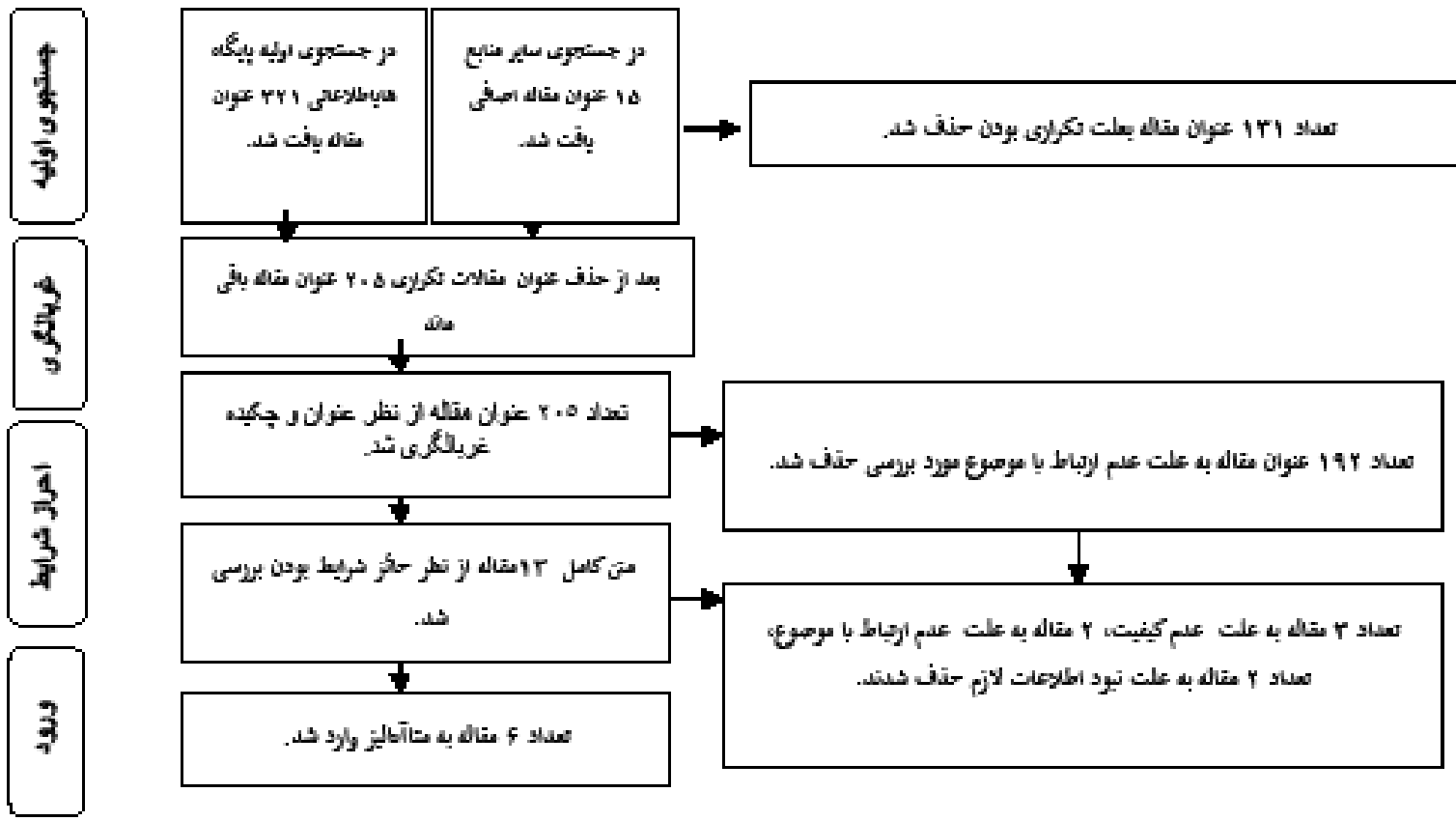

il

و وارد مطالعه شوند. جنانجه اختلافنظرى بين محققان در

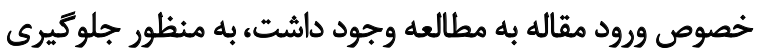

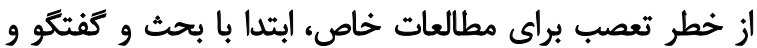

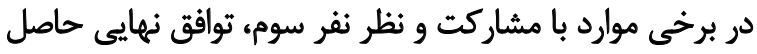

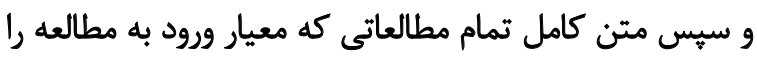

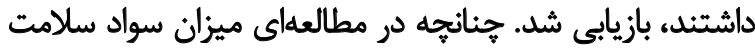

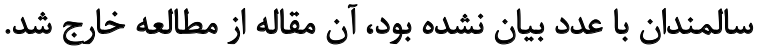

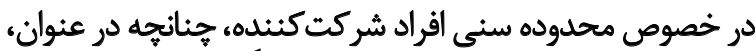

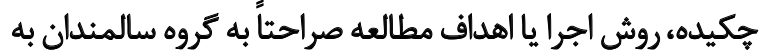

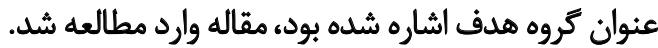

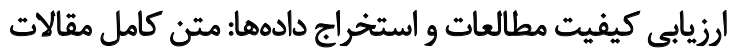

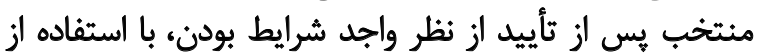

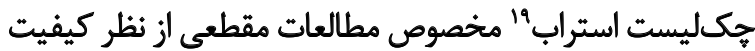

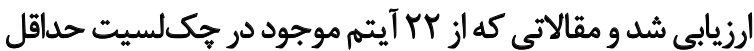

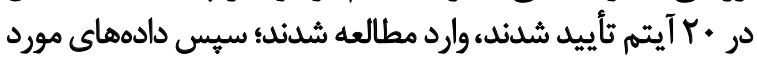

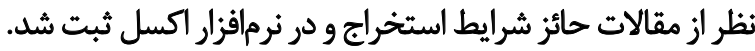

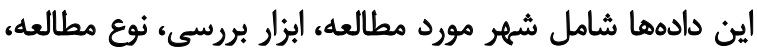

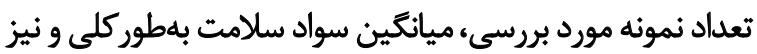
به تفكيك جنس (در صورت وجود كزارش) بوديا

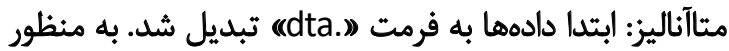

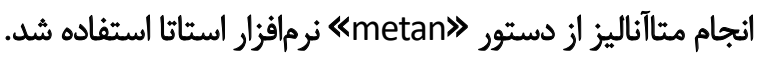
براى بررسى تورش انتشار نمودار قيفى با در نظر كرفتن متغير استاني

\section{STROBE}

تصوير ا. مراحل انتخاب و ورود مطالعات به متأآليز سواد سلامت سالمندان ايرانى

تمامى مطالعات مرتبط، منابع مقالاتى كه معيار ورود به مطالعه

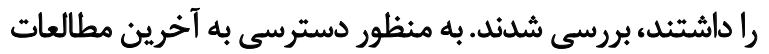

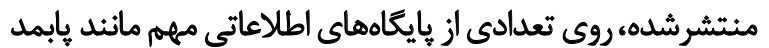

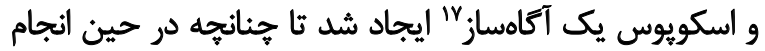

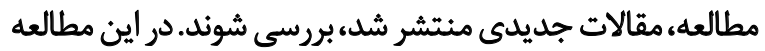
استراترىى جستوجو، غربال

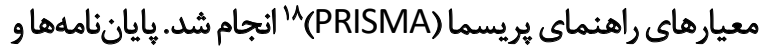

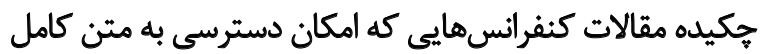
آنها وجود نداشت، از مطالعه خارج شدندي

معيارهاي ورود و خروج و انتخاب مطالعات: ازآنجاكه هدف از

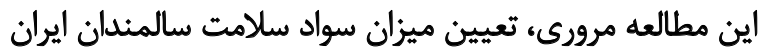

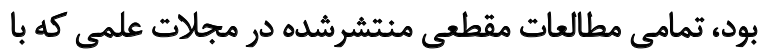

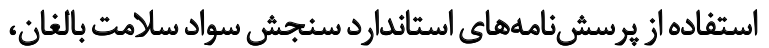

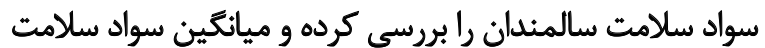

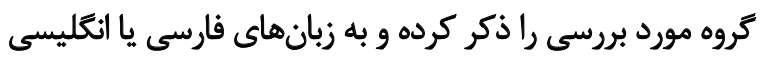

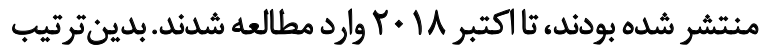

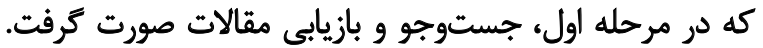

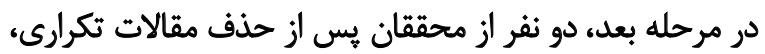

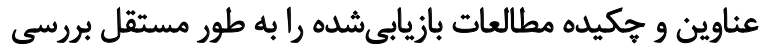

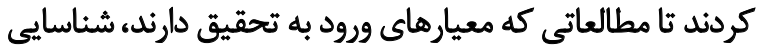

\section{Alert}

18. Preferred Reporting Items for Systematic Reviews and Meta-analyses 
جدول ا. اطلاعات مطالعات منتخب و واردشده به مثآآناليز در مطالعه سواد سلامت سالمندان ايرانى

\begin{tabular}{|c|c|c|c|c|c|c|c|c|}
\hline حجم نمونه & يرسش نامه & جنس - - & (محدوده سني، سالر) & شهر & نو & أنتشارل & نويسنده & رديف \\
\hline س.r برد، سها & TOHFLA & هردان و زنان (جداكانه) & شهرى (91-8. & اصفهان & مغطعى & $r \cdot I r$ & جواد زاده & 1 \\
\hline | & TOHFLA & (بلون تفكيك) & شهرى (بالاتر از •ع) & اصفهلن & مقطعى & $r+1 r$ & رئيسى & r \\
\hline VاI نفر & TOHFLA & (بلون تفونيك) & شهرى (بالاتر از هه) & رفسنجان & مقطعى & $r+18$ & حسيثى & $r$ \\
\hline هو هردة هـ ازن & TOHFLA & هردان و زنان (جداكانه) & شهرى (ND-DA & زاهدان & متطعي & $r \cdot 18$ & انصارى & r \\
\hline V ال مرد، سF If & TOHFLA & هردان و زنان (جداكانه) & 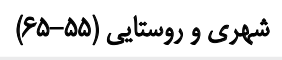 & ايلام & هغطعى & $r+i r$ & برجى & $\Delta$ \\
\hline re. & S-TOHFLA & فقط زنان & شهرى (بالاتر از •ع) & هشهد & هقطعى & $r+1 /$ & مهدى زاده & 8 \\
\hline
\end{tabular}

记

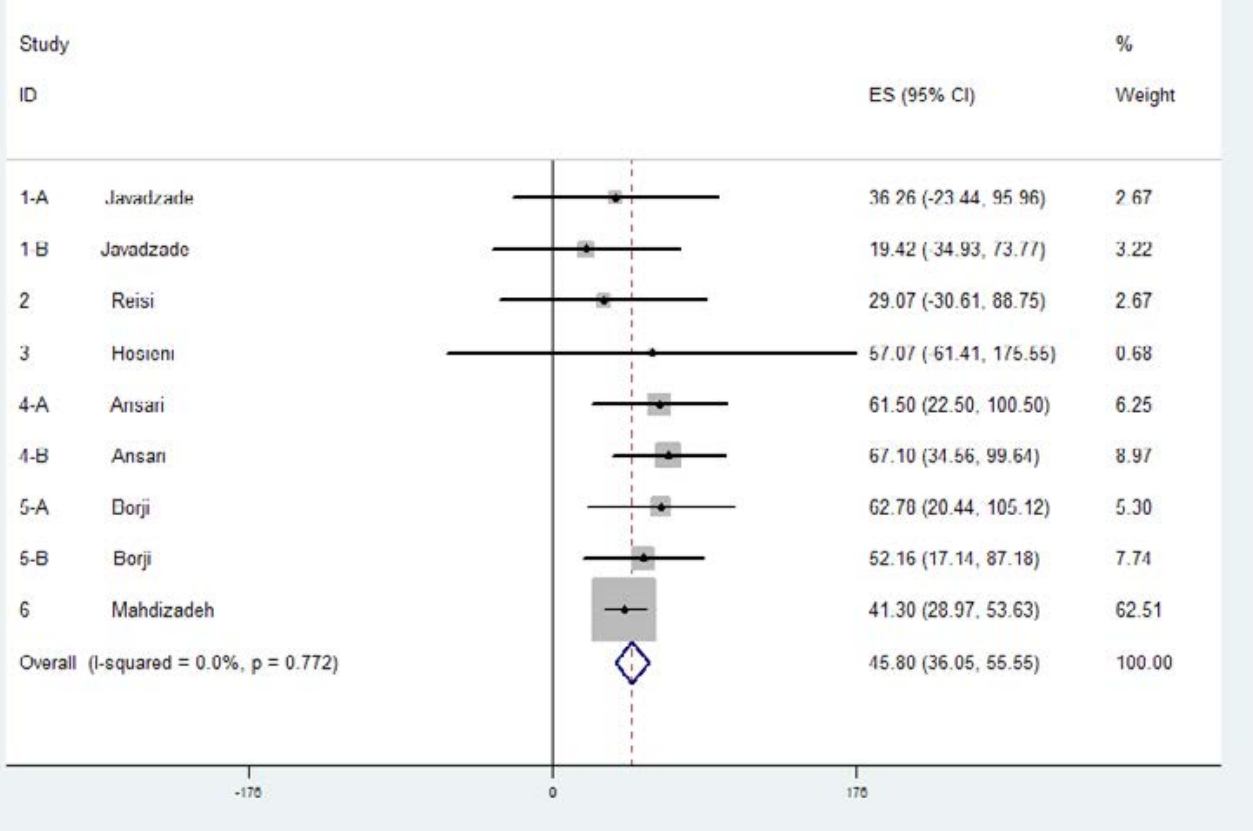

\section{ill}

تصوير r. نمودار انباشت ميانكين نمرات سواد سلامت در مطالعه سواد سلامت سالمندان ايرائى

ع مقاله وارد مرور سيستماتيك و مثتآناليز شد. زمان انتشار اين

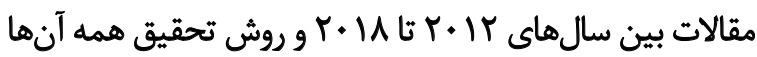

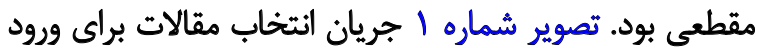

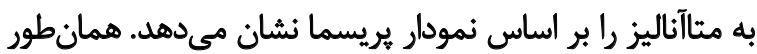

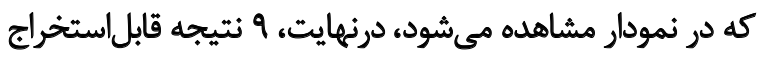

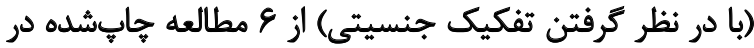

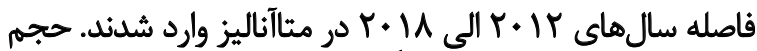

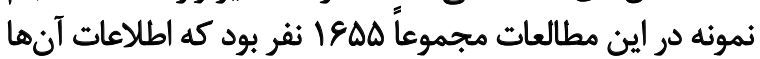
تحليل شد . براى سنجش سواد سلامت در همه مطالعات از ابزار

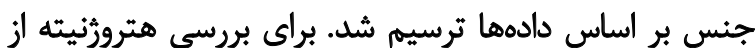

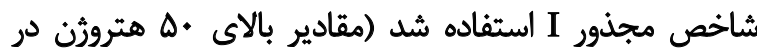

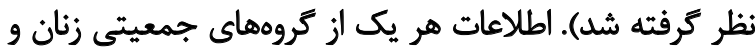

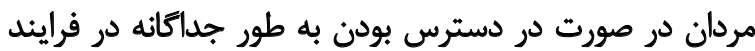

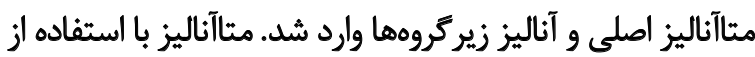
روش اثرات ثابت صورت كرفت و براى هر تحليل نمودار انباشت جداكانهاى ترسيم شد.

L.dت يس از بررسى مقالات بازيابىشده و ارزيابى كيفيت مقالات، 


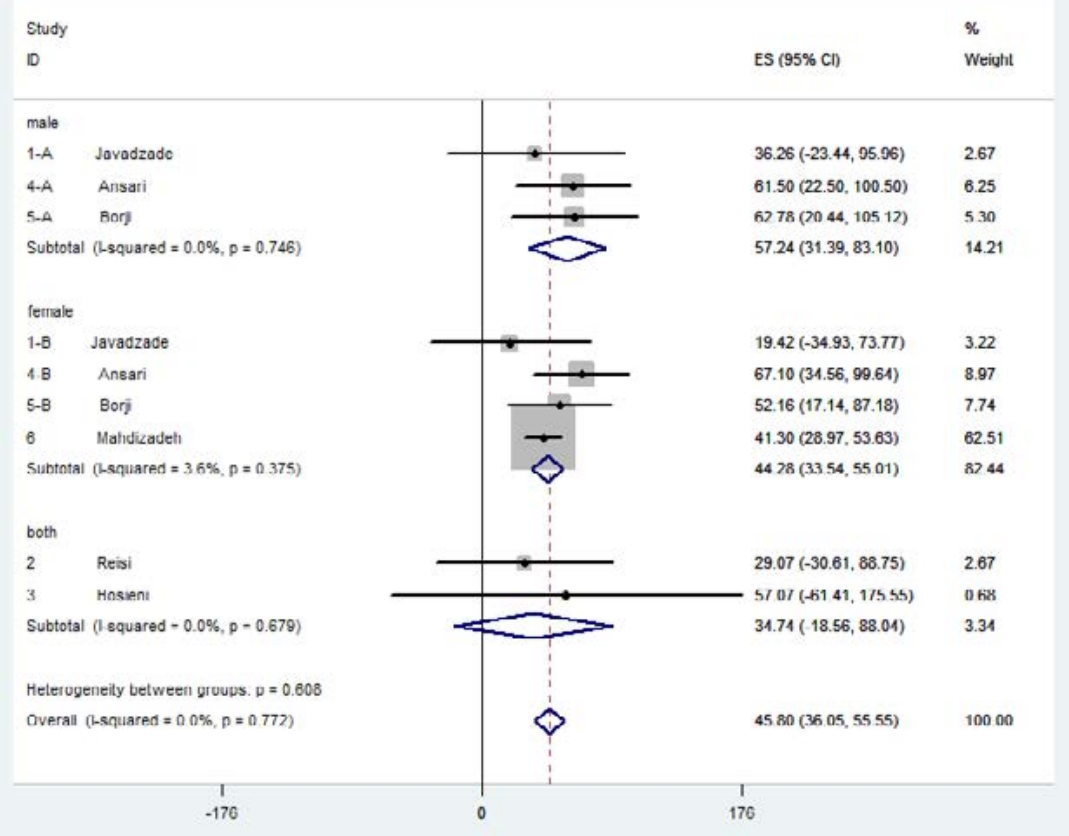

记

تصوير ب. نمودار انباشت ميانكين نمرات سواد سلامث به تفكيك جنس در مطالعه سواد سلامت سالمندان ايرائى

يك كاسهشده به همراه هو درصد فاصله اطمينان نمرات

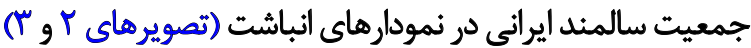

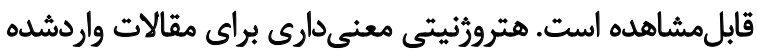

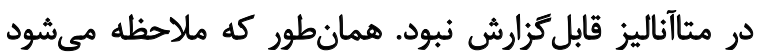

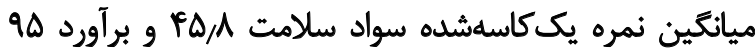

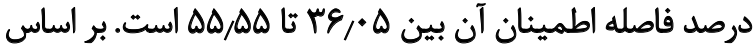

22. The pooled mean scores with the $\mathrm{Cl}$ of $95 \%$
TOFHLA

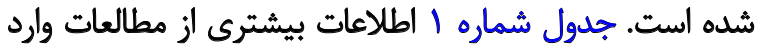

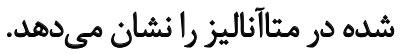
ميانكين نمرات سواد سلامت در هريك از مطالعات و نيز نمره

20. STATA-11.2 (Copyright 1985-2009)

21. The test of functional health literacy in adults

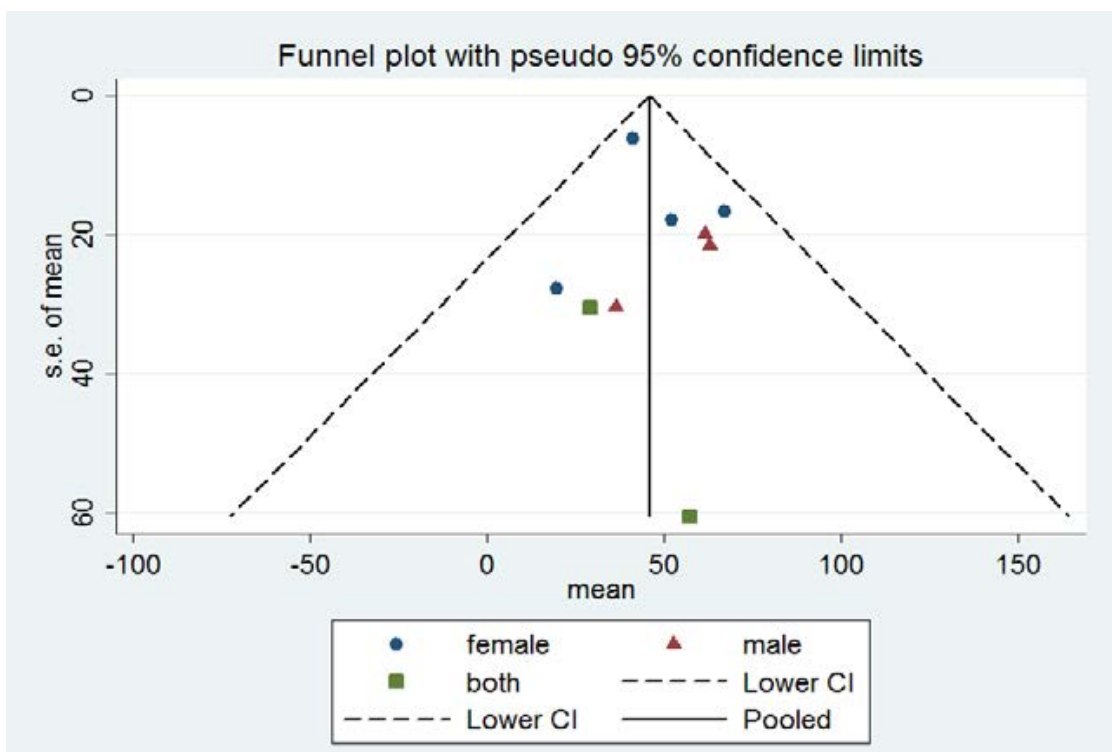


را در حد بايينتر از مردان ارزيابي كرده بودئد؛ اما نتايج نشان

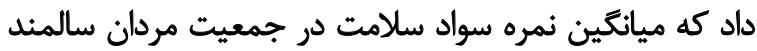

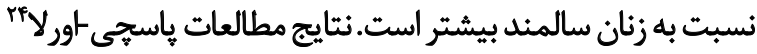

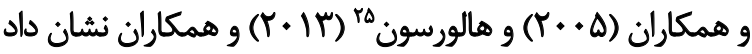

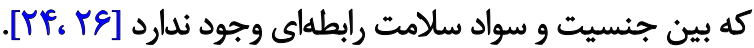

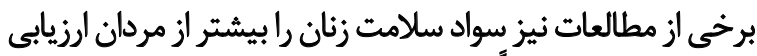

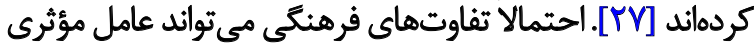
در سطح سواد سلامت زنان ايرانى باشد. همجينين نتايج مطالعه حاضر نشان داد كه سالمندان با سطح

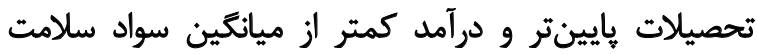

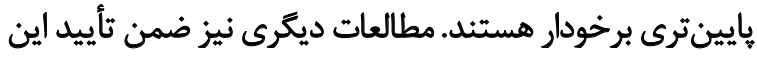

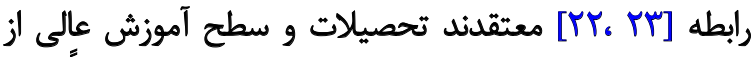

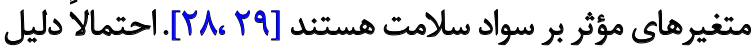
اين رابطه اين است كه افراد با سطح تحصيلات باد بالاتر در خواند

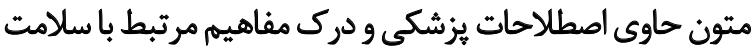

$$
\text { كمتر دجار مشكل مىشوند. }
$$

بر اساس مطالعات قبلى، سواد سلامت بايين مى تواند به طور

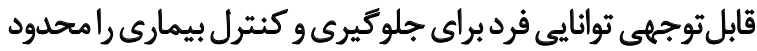

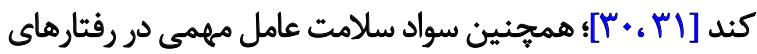

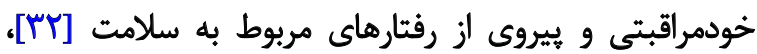

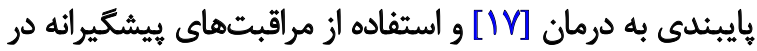

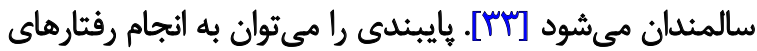

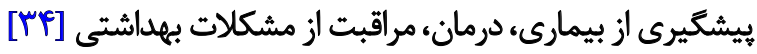

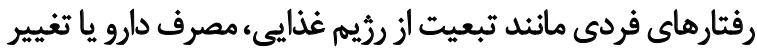

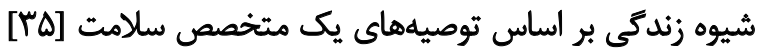

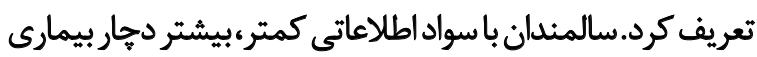

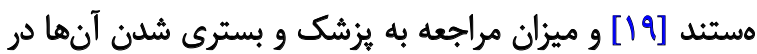

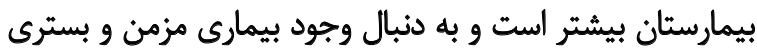

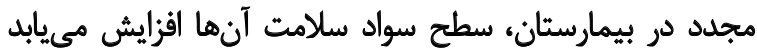

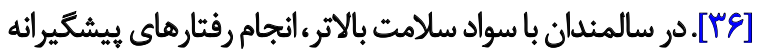

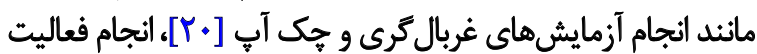

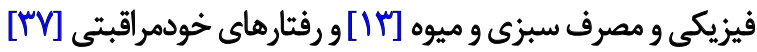

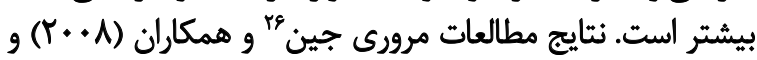

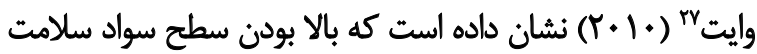

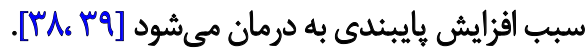

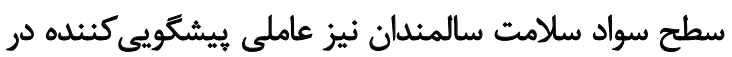

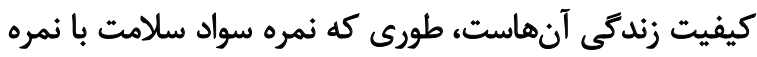

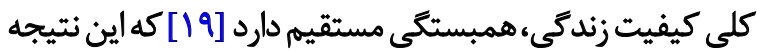

24. Paasche-orlow

25. Halverson

26. Jin

27. Witte
نتايج تكرار مثاناليز در زيركروهها (بر اساس دادههاى تفكيكشده

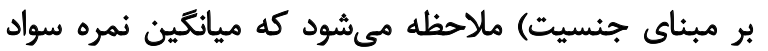

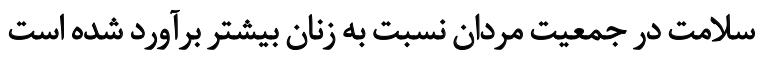

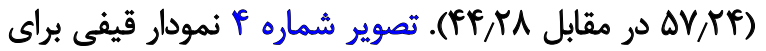

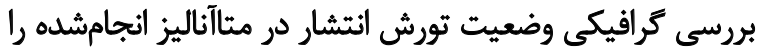

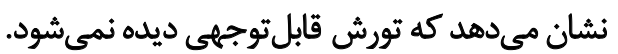

بـ

يس از جستوجو و در نظر كرفتن معيارهاى ورود و خروج

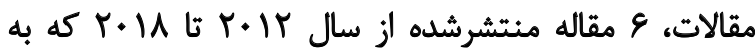

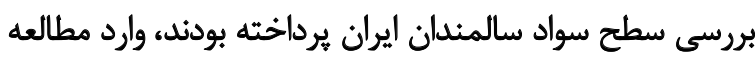

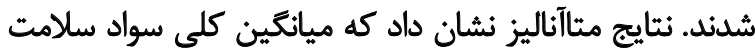

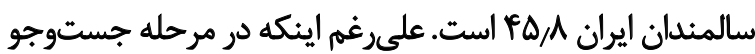

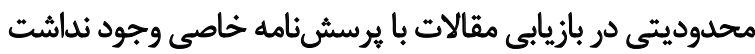

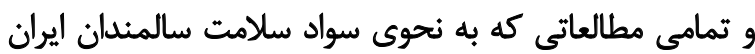

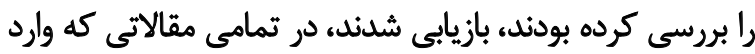

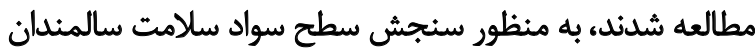

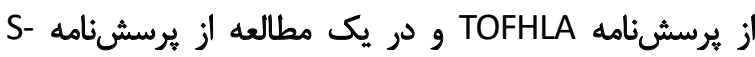

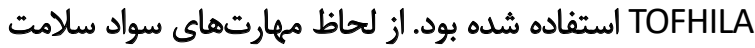

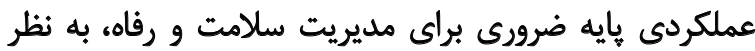

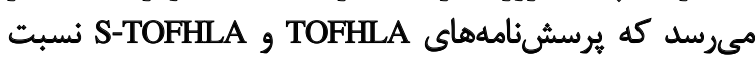

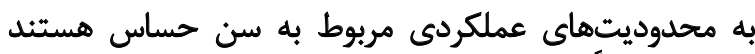

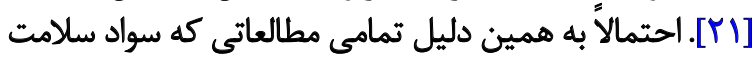

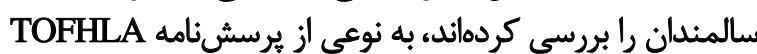

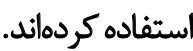

بر اساس نتايج اين مطالعه، سواد سلامت سالمندان ايران در

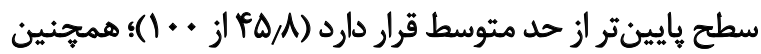

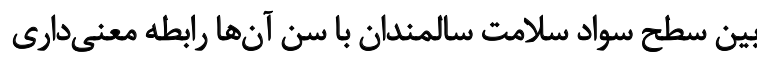

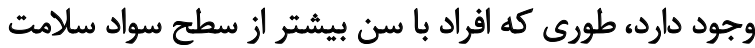

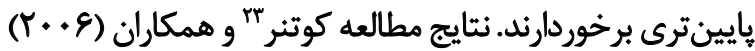

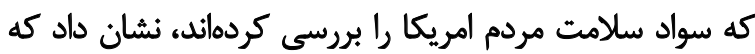

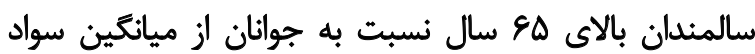

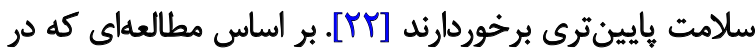

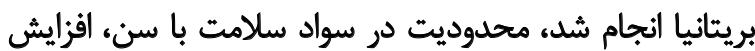

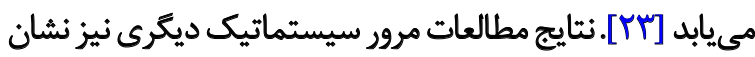

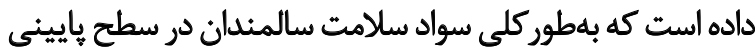

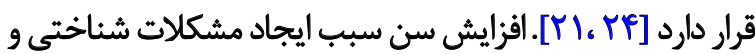

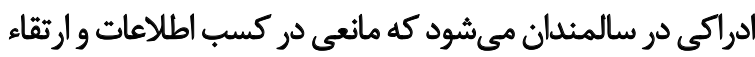

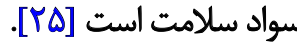

در اين مطالعه از \& مقاله مورد بررسى، يكى مقاله سواد سلامث

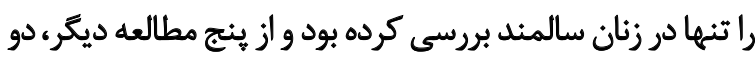

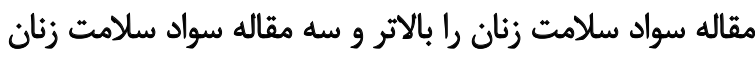

23. Kutner 
است؛ بنابراين به متخصصان و محققان حوزه سالمندى بيشنهاد إنداد

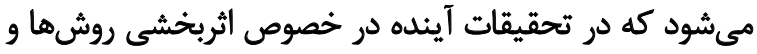

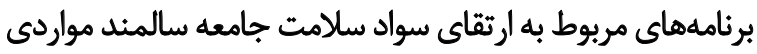

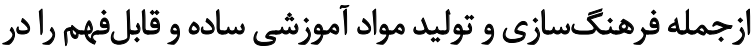
نظر داشته باشـند.

ملاحظات اخلاقى يبيروى از اصول اخلاق يُوهش

اين مطالعه در كميته اخلاق دانشكاه علوم يزشكى سمنان با كد (IR.SEMUMS.REC.1397.045) تصويب شداف

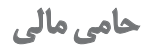

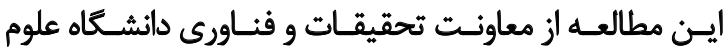
يزشـكى سـمنان مـورد حمايست مالـى دريافت كرده اسـت.

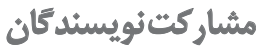

تمــــام نويســــندكان در آماده سـازى اين مقاله مشـاركث داشــته اند.

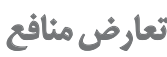
هيج تعارض منافعى بين نويسندكان وجود ندارد.

$$
\text { تشكرو وقدروائي }
$$

اين مطالعه حاصل طرح بيروهشى مصوب دانشكاه علوم

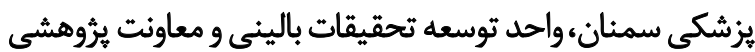

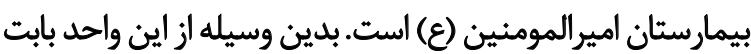

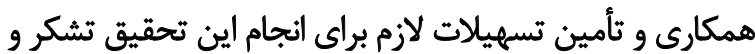

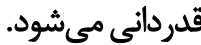

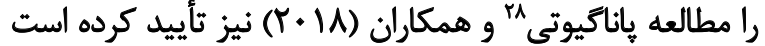

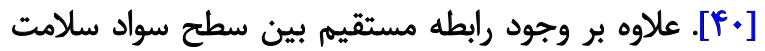

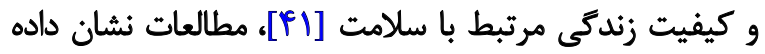

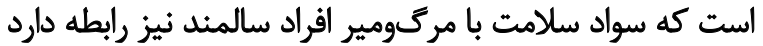

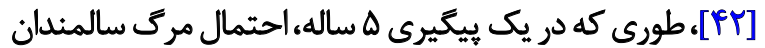

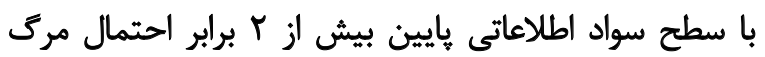

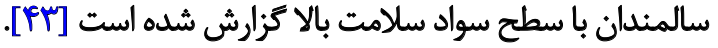

مههمترين نقطهقوت اين مطالعه اين است كه اولين مطالعه

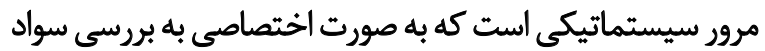

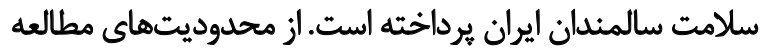

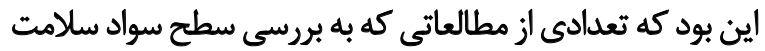

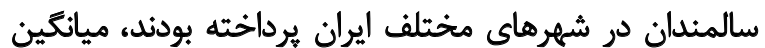

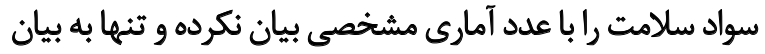

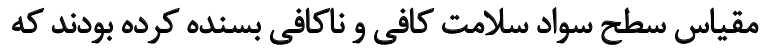

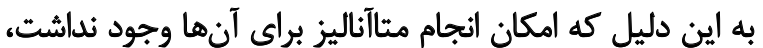
وارد مطالعه نشُدند.

محدوديتهاى مربوط به جستوجوى تركيبى در پإيكاههاى

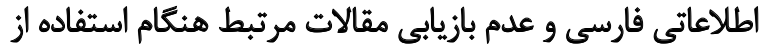

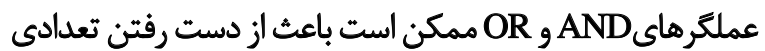

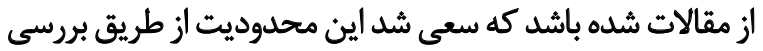

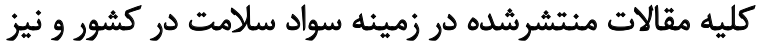

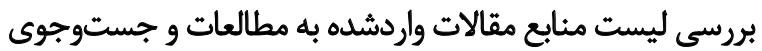

$$
\text { دستى يوشش داده شود. }
$$

\section{تتيجهيَيرىنهايى}

نتايج اين مطالعه نُشان داد كه سالمندان ايرانى سواد سلامت باديت

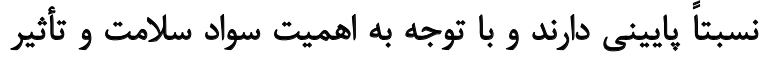

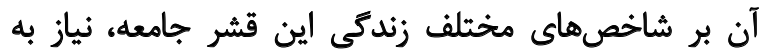

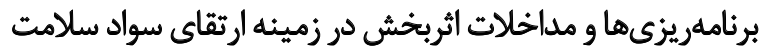

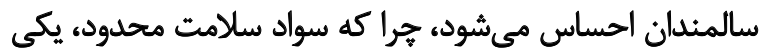

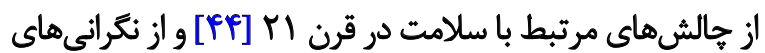

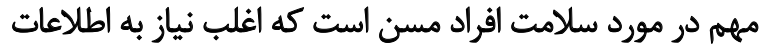

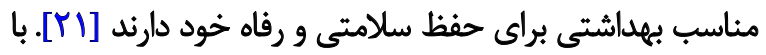

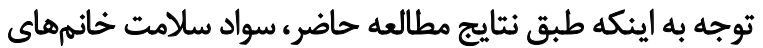

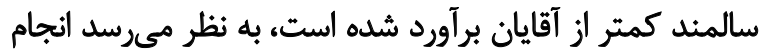

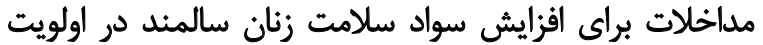

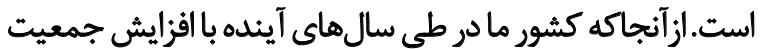

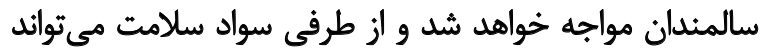

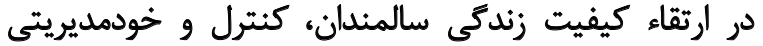

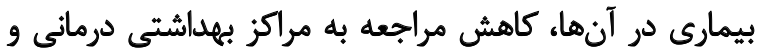

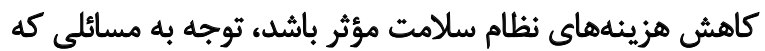

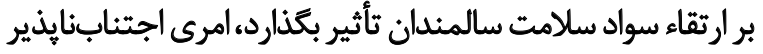

28. Panagioti 


\section{References}

[1] Fransen M, Van Schaik T, Twickler T, Essink-Bot M. Applicability of internationally available health literacy measures in the Netherlands. Journal of Health Communication. 2011; 16(sup3):134-49. [DOI:10.1080/10810730.2011.604383] [PMID]

[2] Berkman ND, Davis TC, McCormack L. Health literacy: What is it? Journal of Health Communication. 2010; 15(S2):9-19. [DOI :10.1080/10810730.2010.499985] [PMID]

[3] Social Determinants of Health. Commission on Social Determinants of Health final report. Geneva: World Health Organization; 2008.

[4] Matlabi H, Shaghaghi A, Amiri S. A pilot physical activity initiative to improve mental health status amongst Iranian institutionalized older people. Health Promotion Perspectives. 2014; $4(1): 68$.

[5] Hajduchova H, Bartlova S, Brabcova I, Sedova L, Tothova VS Health Literacy Of The Elderly. $3^{\text {rd }}$ International Multidisciplinary Scientific Conference on Social Sciences and Arts. 2016 Sep 24-30. Albena: Bulgaria

[6] Ferguson B. Health literacy and health disparities: The role they play in maternal and child health. Nursing for Women's Health. 2008; 12(4):286-98. [DOI:10.1111/j.1751486X.2008.00343.x] [PMID]

[7] Peerson A, Saunders M. Health literacy revisited: What do we mean and why does it matter? Health promotion International. 2009; 24(3):285-96. [DOI:10.1093/heapro/dap014] [PMID]

[8] Morovatisharifabad M, Ghofranipour F, Heydarnia A, Babaeirochi G. Perceived religious support of health promoting behavior and status doing these behaviors in aged 65 years and older in Yazd. The Journal of Shahid Sadoughi University of Medical Sciences. 2004; 12(1):23-9.

[9] Berens E, Ganahl K, Vogt D, Schaeffer D. Health literacy among elderly immigrants in Germany. Findings from a crosssectional survey. European Journal of Public Health. 2018; 28:191-2. [DOI:10.1093/eurpub/cky048.245]

[10] Liu YB, Xue LL, Xue HP, Hou P. Health literacy, self-care agency, health status and social support among elderly Chinese nursing home residents. Health Education Journal. 2018; 77(3):303-11. [DOI:10.1177/0017896917739777]

[11] La Vonne AD, Zun LS. Assessing adult health literacy in urban healthcare settings. Journal of the National Medical Association. 2008; 100(11):1304-8. [DOI:10.1016/S00279684(15)31509-1]

[12] Lee SS, Choi YS, Lee DS, Nam SH. A study on the health literacy of the elderly in rural area. Biomedical Research-India. 2017; 28(17):7567-73.

[13] Reisi M, Javadzade SH, Heydarabadi AB, Mostafavi F, Tavassoli E, Sharifirad G. The relationship between functional health literacy and health promoting behaviors among older adults. Journal of Education and Health Promotion. 2014; 3:119. [DOI:10.4103/2277-9531.145925] [PMID] [PMCID]

[14] Ansari H, Almasi Z, Ansari-Moghaddam A, Mohammadi M, Peyvand M, Hajmohammadi M, et al. Health literacy in older adults and its related factors: A cross-sectional study in Southeast Iran. Health Scope. 2016; 5(4):e37453. [DOI:10.17795/ jhealthscope-37453]
[15] Mohseni M, Khanjani N, Iranpour A, Tabe R, Borhaninejad VR. The relationship between health literacy and health status among elderly people in Kerman. Iranian Journal of Ageing. 2015; 10(2):146-55.

[16] Fathi F, RezaeeSofi M. The relationship between health literacy and physical activity level of elderly women in the city of Urmia. Journal of Health Literacy. 2017; 2(1):12-21. [DOI:10.18869/acadpub.jhl.2.1.12]

[17] Kooshyar H, Shoorvazi M, Dalir Z, Hosseini M. Health literacy and its relationship with medical adherence and health-related quality of life in diabetic community-residing elderly. Journal of Mazandaran University of Medical Sciences. 2014; 23(1):134-43.

[18] Knussen C, Tolson D, Brogan CA, Swan IR, Stott DJ, Sullivan F. Family caregivers of older relatives: Ways of coping and change in distress. Psychology, Health and Medicine. 2008; 13(3):274-90. [DOI:10.1080/13548500701405483] [PMID]

[19] Hosieni F, Mirzaei T, Ravari A, Akbary A. The relationship between health literacy and quality of life in retirement of Rafsanjan University of Medical Sciences. Journal of Health Literacy. 2016; 1(2):92-9.

[20] Javadzade SH, Sharifirad G, Radjati F, Mostafavi F, Reisi M, Hasanzade A. Relationship between health literacy, health status, and healthy behaviors among older adults in Isfahan, Iran. Journal of Education and Health Promotion. 2012;1. [DOI:10.4103/22779531.100160] [PMID] [PMCID]

[21] Kobayashi LC, Wardle J, Wolf MS, von Wagner C. Aging and Functional Health Literacy: A Systematic Review and Meta-Analysis. Thejournals of Gerontology Series B, Psychological Sciences and Social Sciences. 2016; 71(3):445-57. [DOI:10.1093/geronb/ gbu161] [PMID] [PMCID]

[22] Kutner M, Greenburg E, Jin Y, Paulsen C. The health literacy of America's Adults: Results from the 2003 National Assessment of Adult Literacy. NCES 2006-483. Washington, DC: National Center for Education Statistics; 2006

[23] Wagner CV, Knight K, Steptoe A, Wardle J. Functional health literacy and health-promoting behaviour in a national sample of British adults. Journal of Epidemiology and Community Health. 2007; 61(12):1086. [DOI:10.1136/jech.2006.053967] [PMID] [PMCID]

[24] Paasche-Orlow MK, Parker RM, Gazmararian JA, NielsenBohlman LT, Rudd RR. The Prevalence of Limited Health Literacy. Journal of General Internal Medicine. 2005;20(2):175-84 [DOI:10.1111/j.1525-1497.2005.40245.x] [PMID] [PMCID]

[25] Nguyen HT, Kirk JK, Arcury TA, Ip EH, Grzywacz JG, Saldana SJ, et al. Cognitive function is a risk for health literacy in older adults with diabetes. Diabetes Research and Clinical Practice. 2013; 101(2):141-7. [DOI:10.1016/j.diabres.2013.05.012] [PMID] [PMCID]

[26] Halverson J, Martinez-Donate A, Trentham-Dietz A, Walsh MC, Strickland JS, Palta M, et al. Health literacy and urbanicity among cancer patients. The Journal of rural health : official journal of the American Rural Health Association and the National Rural Health Care Association. 2013; 29(4):392-402. [DOI:10.1111/ jrh.12018] [PMID] [PMCID]

[27] MacLeod S, Musich S, Gulyas S, Cheng Y, Tkatch R, Cempellin $\mathrm{D}$, et al. The impact of inadequate health literacy on patien satisfaction, healthcare utilization, and expenditures among older adults. Geriatric Nursing (New York, NY). 2017; 38(4):334-41 [DOI:10.1016/j.gerinurse.2016.12.003] [PMID] 
[28] Sorlie V, Lopez RA. When language, health literacy, and miscommunication collide: Tremors versus seizures. Family medicine. 2011; 43(1):48-50.

[29] Cho YI, Lee S-YD, Arozullah AM, Crittenden KS. Effects of health literacy on health status and health service utilization amongst the elderly. Social Science \& Medicine. 2008; 66(8):1809-16. [DOI:10.1016/j.socscimed.2008.01.003] [PMID]

[30] Schillinger D, Grumbach K, Piette J, Wang F, Osmond D, Daher $\mathrm{C}$, et al. Association of health literacy with diabetes outcomes. Journal of the American Medical Association. 2002; 288(4):475-82. [DOI:10.1001/jama.288.4.475] [PMID]

[31] Marshall S, Sahm L, McCarthy S. Health literacy in Ireland: reading between the lines. Perspectives in Public Health. 2012; 132(1):31-8. [DOI:10.1177/1757913911431034] [PMID]

[32] Chen AM, Yehle KS, Albert NM, Ferraro KF, Mason HL, Murawski MM, et al. Relationships between health literacy and heart failure knowledge, self-efficacy, and self-care adherence. Research in Social \& Administrative Pharmacy. 2014; 10(2):378-86. [DOI:10.1016/j.sapharm.2013.07.001] [PMID] [PMCID]

[33] Chen JZ, Hsu HC, Tung HJ, Pan LY. Effects of health literacy to self-efficacy and preventive care utilization among older adults. Geriatrics \& Gerontology International. 2013; 13(1):70-6. [DOI:10.1111/j.1447-0594.2012.00862.x] [PMID]

[34] Paasche-Orlow MK, Wolf MS. The causal pathways linking health literacy to health outcomes. American Journal of Health Behavior. 2007; 31(1):S19-S26. [DOI:10.5993/AJHB.31.s1.4]

[35] Sabaté E. Adherence to long-term therapies: Evidence for action: Geneva: World Health Organization; 2003.

[36] Borji M, Tarjoman A, Otaghi M, Salimi E, Naseri A. Health literacy level and its related factors among the elderlies in Ilam in 2015. Iran Journal of Nursing. 2017; 30(108):33-43. [DOI:10.29252/ ijn.30.108.33]

[37] Mahdizadeh M, Solhi M. Relationship between self-care behaviors and health literacy among elderly women in Iran, 2015. Electron Physician. 2018; 10(3):6462-9. [DOI:10.19082/6462] [PMID] [PMCID]

[38] Jin J, Sklar GE, Min Sen Oh V, Chuen Li S. Factors affecting therapeutic compliance: A review from the patient's perspective. Therapeutics and Clinical Risk Management. 2008; 4(1):269-86. [DOI:10.2147/TCRM.S1458] [PMID] [PMCID]

[39] Witte PG. Health Literacy: Can We Live without It? Adult Basic Education and Literacy Journal. 2010; 4(1):3-12.

[40] Panagioti M, Skevington SM, Hann M, Howells K, Blakemore A, Reeves D, et al. Effect of health literacy on the quality of life of older patients with long-term conditions: A large cohort study in UK general practice. Quality of Life Research. 2018; 27(5):1257-68. [DOI:10.1007/s11136-017-1775-2] [PMID] [PMCID]

[41] Park NH, Song MS, Shin SY, Jeong J-h, Lee HY. The effects of medication adherence and health literacy on health-related quality of life in older people with hypertension. International Journal of Older People Nursing. 2018; 13(3):e12196. [DOI:10.1111/ opn.12196] [PMID]

[42] Smith SG, Jackson SE, Kobayashi LC, Steptoe A. Social isolation, health literacy, and mortality risk: Findings from the English longitudinal study of ageing. Health Psychology. 2018; 37(2):160-9. [DOI:10.1037/hea0000541] [PMID] [PMCID]
[43] Bostock S, Steptoe A. Association between low functional health literacy and mortality in older adults: Longitudinal cohort study. The BMJ. 2012; 344. [DOI:10.1136/bmj.e1602] [PMID] [PMCID]

[44] Hälleberg Nyman M, Nilsson U, Dahlberg K, Jaensson M. Association between functional health literacy and postoperative recovery, health care contacts, and health-related quality of life among patients undergoing day surgery: Secondary analysis of a randomized clinical trial. JAMA Surgery. 2018; 153(8):738-45. [DOI:10.1001/jamasurg.2018.0672] [PMID] [PMCID] 